\title{
La Biología Evolucionaria Desenvolvimiental y el surgimiento de una nueva teoría de la evolución*
}

The Evolutionary Developmental Biology and the Emergence of a New Theory of Evolution

Gustavo Caponi ${ }^{\dagger}$

\begin{abstract}
Resumen
Los resultados de la Biología Evolucionaria Desenvolvimiental presuponen una segunda teoría de la evolución. Una teoría que, ofreciéndose como complementaria, y no como contraria o alternativa, a la Teoría de la Selección Natural, tampoco puede considerarse su mera subalterna o su auxiliar. Esa teoría, sin embargo, no es fácilmente individualizable. Ella está diseminada y tácitamente supuesta en los desarrollos conceptuales y en los resultados empíricos de la Evo-Devo. Pero, si se identifica lo que Stephen Toulmin caracterizaría como el ideal de orden natural al que responden dichos desarrollos y resultados, los contornos y la naturaleza de esa teoría comienzan a hacerse un poco más nítidos.
\end{abstract}

Palabras clave: evo-devo - ideal de orden natural - objetivos explicativos - teoría de la selección natural - Toulmin

\begin{abstract}
The results of Evolutionary Developmental Biology presuppose a second theory of evolution. A theory that, being complementary, and not contrary or alternative, to the Theory of Natural Selection, cannot be considered its mere subaltern or its auxiliary. That theory, however, is not easily individualized. It is disseminated, and tacitly assumed, in the conceptual developments and in the empirical results of Evo-Devo. But if one identifies what Stephen Toulmin would characterize as the ideal of natural order to which these developments and results respond, the outlines and nature of that theory begin to become a little sharper.
\end{abstract}

Keywords: evo-devo - ideal of natural order - explanatory targets - theory of natural selection - Toulmin

* Recibido: 3 de septiembre de 2015. Aceptado con revisiones: 5 de diciembre de 2015.

† Departamento de Filosofía, Universidad Federal de Santa Catarina (UFSC), Brasil. Para contactar al autor, por favor, escribir a: gustavoandrescaponi@gmail.com.

Metatheoria 6(2)(2016): 65-80. ISSN 1853-2322. eISSN 1853-2330.

(C) Editorial de la Universidad Nacional de Tres de Febrero. Publicado en la República Argentina. 
L'objet d'un vrai critique devrait être de découvrir quel problème l'auteur (sans le savoir ou le sachant) s'est posé, et de chercher s'il l'a résolu ou non. Paul Valery, Tel quel.

\section{Presentación}

Entre en 1940 y 1990, cuando el devenir de la Biología Evolucionaria estaba pautado por la hegemonía neodarwiniana, la respuesta a la pregunta por cuál era la teoría de la evolución no podía ser otra que "la Teoría de la Selección Natural". Se podía discutir cuál era su formulación exacta y cuáles sus supuestos, cuáles eran las hipótesis auxiliares que ella debía y podía aceptar para cumplir con sus objetivos explicativos y cuáles otras teorías biológicas, siempre consideradas como sus posibles subalternas, eran compatibles con ella; pero, lo que sólo algunos pocos outsiders se atrevían a cuestionar era el hecho de que esa teoría definía las coordenadas en la que los fenómenos evolutivos debían situarse para ser explicados. Esa situación, sin embargo, podría estar cambiando. Los desarrollos de la Biología Evolucionaria Desenvolvimiental, ${ }^{1}$ la llamada Evo-Devo, que desde la década de los noventa, vienen agitando el por mucho tiempo calmo horizonte teórico de la Biología Evolucionaria, parecen presuponer una segunda teoría de la evolución. Una teoría que, ofreciéndose como complementaria, y no como contraria o alternativa, a la Teoría de la Selección Natural, tampoco puede considerarse su mera subalterna o su auxiliar.

Esa teoría, claro, no es fácilmente individualizable. Como ocurre en general con las teorías biológicas y como ocurrió inicialmente con la propia Teoría de la Selección Natural, la misma carece todavía de una formulación totalmente explícita y sistemática. Ella está, por el contrario, diseminada y tácitamente supuesta en los desarrollos conceptuales y en los resultados empíricos de la Evo-Devo. Pero, si prestamos atención a los problemas que esos desarrollos y esos resultados pretenden resolver, podremos ver que se trata de problemas que pueden ser planteados, y resueltos, con relativa independencia de la propia Teoría de la Selección Natural. Se trata, por decirlo de otro modo, de problemas que responden a preguntas que esta última teoría no sólo no procura, ni precisa, responder, sino que además tampoco permite plantear; y esa diferencia de objetivos explicativos, según espero poder mostrar aquí, no es una simple diferencia pragmática, no es una simple diferencia de intereses. La misma obedece al hecho de que la Biología Evolucionaria Desenvolvimiental responde a lo que, siguiendo a Stephen Toulmin (1961, pp. 44 y ss.), caracterizaré como un ideal de orden natural diferente de aquel presupuesto por la Teoría de la Selección Natural.

Para Toulmin (1961, p. 45), los ideales de orden natural son presupuestos que, para una teoría particular, definen lo que es el caso cuando nada ocurre y así establecen el horizonte de permanencia sobre el cual irrumpen los hechos a ser explicados por dicha teoría. Un ideal de orden natural, podríamos decir, define el estado o el devenir de las cosas que, dada una determinada teoría, se considera obvio, necesario, natural, de por sí comprensible, y, por eso, carente de toda necesidad de explicación. Siendo precisamente el desvío o la ruptura de ese orden ideal, y nunca real, lo que aparecerá como merecedor de las explicaciones que, a partir de esa teoría, se pueda llegar a formular. Pienso, por eso, que la individualización de un ideal de orden natural puede constituir un buen recurso para establecer la

\footnotetext{
${ }^{1}$ No hay duda que al traducir "Evolutionary Developmental Biology” por "Biología Evolucionaria Desenvolvimiental” corrí algunos riesgos. El más obvio, pero ni el primero ni el más importante, es haber traducido “developmental" por "desenvolvimiental”. Al hacerlo me basé en el hecho de que, aun en desuso, la expresión "desenvolvimiento", que continúa siendo sinónima de "desarrollo", permite construir adjetivos análogos a funcional u organizacional. La expresión "desarrollo", en cambio, no lo permite. Pero he ahí en donde reside el más serio, y el primero, de los riesgos corridos: la expresión "Evolutionary Developmental Biology" debería y podría ser fácilmente traducida por "Biología Evolucionaria del Desarrollo". En ella "evolutionary" está adjetivando a "developmental" y no a la inversa como en "Biología Evolucionaria Desenvolvimiental". Considero, sin embargo, que esta última expresión, al igual que la francesa "Biologie Développementale de l'Évolution" (Schmitt 2006, p. 431), expresa mejor el hecho de que esta nueva disciplina no es otra cosa que un capítulo de la Biología Evolucionaria calificado o instruido por la perspectiva desenvolvimiental.
} 
existencia y los perfiles más generales de una teoría, aun cuando ésta no haya sido todavía plenamente articulada y explícitamente formulada por aquellos que de hecho la están aplicando.

Es decir: una teoría puede ser identificada y reconstruida, o por lo menos entrevista, partiendo de una correcta elucidación del ideal de orden natural que le da sentido; y eso es lo que aquí intentaré hacer con esa segunda teoría de la Biología Evolucionaria que, según digo, está siendo edificado por los cultores de la Evo-Devo. Mi tesis es que, así como la permanencia de la forma ancestral primitiva puede ser considerada como el ideal de orden natural de la Teoría de la Selección Natural (Caponi 2004); la postulación de un morfoespacio virtual regularmente ocupado, definiría el ideal de orden natural de la Biología Evolucionaria Desenvolvimiental. Por eso, mientras la Teoría de la Selección Natural nos provee los recursos para explicar la razón de ser de cada ocupación del morfoespacio que se produce con cada innovación o invención morfológica producida por la evolución, la Biología Evolucionaria Desenvolvimiental pretende darnos a conocer los factores que, con independencia de la selección natural, explican el hecho de que esa ocupación del morfoespacio sea sesgada o irregular.

\section{La navaja de Darwin}

Como ejemplo paradigmático de ideal del orden natural, Toulmin (1961, p. 56) nos propone al "Principio de Inercia": todo cuerpo continúa en estado de reposo, o de movimiento rectilineo y uniforme, a menos que sea compelido a cambiar dicho estado de movimiento por aplicación de una fuerza. Esta primera ley de Newton nos dice, en efecto, que la permanencia de un cuerpo en cualquiera de esos dos estados es lo esperable, lo normal, lo natural. Lo que debe ser explicado, por lo tanto, es la salida del reposo o la salida del movimiento rectilíneo uniforme; y toda la física newtoniana nos ofrece el modo de explicar y calcular los desvíos de ese estado en virtud de ciertas fuerzas y leyes adicionales como, por ejemplo, la Ley de Gravitación.

Aunque ese principio hoy nos resulte obvio, desde su aparente trivialidad, él define, al mismo tiempo, cómo son las cosas cuando nada ocurre, qué significa que algo ocurra y cuál debe ser la naturaleza de la causa de ese acontecimiento que ocurre. Si un cuerpo está en reposo o en movimiento rectilíneo uniforme; entonces, nada ocurre, nada debe ser explicado. Pero, si él se desvía de ese estado, la teoría define el repertorio de fuerzas que nos permitirían, no solamente explicar y prever ese desvío; sino también calcular su magnitud y su sentido. Las indagaciones deberán versar, entonces, sobre el modo en que ese repertorio de fuerzas habrá de utilizarse, y eventualmente ampliarse o modificarse, para así poder construir esas explicaciones y previsiones.

Pero, al igual que otros aspectos de la gramática científica, los ideales de orden natural son regionales: diferentes teorías científicas, al interior de diferentes dominios disciplinares, obedecen a ideales diferentes; y es en este sentido que puede decirse que el movimiento rectilíneo uniforme de la Teoría de la Selección Natural, aquello que para ella constituye el estado natural de las cosas, su ideal de orden natural, es siempre la permanencia de la forma ancestral común; y es el alejamiento de esta forma ancestral aquello que, en cada caso particular, debe ser explicado. Si las especies no cambiasen y no se diversificasen, nada habría para explicar; pero, en la medida en que eso ocurra, como de hecho parece que ocurre, será preciso preguntar ¿por qué? Es decir: ¿por qué existen toda esa diversidad y ese cambio y no más bien la permanencia de la forma originaria? O para citar un ejemplo célebre y concreto: ¿por qué todos esos diseños diferentes de pico de pinzón y no más bien un único diseño?

En la naturaleza darwiniana differentiae non sunt multiplicanda praeter necessitatem; y es en ese sentido que podemos hablar de un principio de parsimonia ontológica que allí funcionaría como ideal de orden natural. Para el darwinismo, en efecto, no hay diferencia que no tenga una razón de ser; y esa razón de ser debe ser encontrada, caso a caso, en base a la Teoría de la Selección Natural. Es ésta, en efecto, la que nos enseña a reconstruir, para cada caso particular, ese balance entre ganancias y pérdidas que se constituye en la razón de ser de cada diferencia. Para cada alejamiento de la forma ancestral debe haber alguna 
explicación que nos muestre que esa diferenciación responde a una presión selectiva que debe ser identificada.

Se me podría objetar, lo sé, que la Teoría de la Selección Natural no sólo busca explicar diferencias: ella también pretende y puede explicar semejanzas. Tal el caso, por ejemplo, de los rasgos análogos. Su presencia en dos o más especies filogenéticamente distantes puede y debe ser explicado por selección natural; y eso parece ir en contra de la alegación de que el objetivo explicativo propio de dicha teoría sea siempre la diferenciación o la divergencia de las formas. Es necesario entender, sin embargo, que la Teoría de la Selección Natural esas analogías son consideradas como el efecto colateral, superficial, del entrecruzamiento accidental de dos procesos independientes de diferenciación cuyos puntos de partida son formas ancestrales distintas.

Pensemos, por ejemplo, en las membranas interdigitales de esa zarigüeya semi-acuática, el Chironectes minimus, que en Brasil es conocida como gambá-d'agua (ver Fernandez et al. 2007). Esta adaptación al nado constituye un rasgo apomorfico comparado con la ausencia de esas membranas que encontramos en las otras especies del género. Especies entre las que, suponemos, también se encontraría el ancestro del Chironectes minimus. Pero, ese mismo rasgo también constituye una homoplasia, una analogía, comparado con las membranas interdigitales de las nutrias. Y, sea en cuanto que apomorfia o en cuanto que homoplasia, ese rasgo podrá ser explicado apelando a la selección natural. En el primer caso, se tratará, claramente, de la explicación de una diferencia; y en el segundo caso se tratará, aparentemente, de la explicación de una semejanza. Sin embargo, si analizamos más detenidamente la cuestión, veremos que la explicación de la semejanza se reduce a la explicación de la diferencia. En este ejemplo, como en cualquier otro ejemplo semejante, la homoplasia quedará reducida a una semejanza accidental producida por el proceso selectivo que generó la apomorfia.

Este último proceso ocurre con total independencia de que la homoplasia ocurra o no: las nutrias podrían no tener membranas interdigitales, o simplemente podrían no existir; pero aun así las presiones selectivas que afectaron al gambá d'agua producirían ese rasgo en él. Y lo mismo podría decirse de las nutrias con relación a las zarigüeyas. Lo que en ellas produjo el rasgo en cuestión es un proceso, tal vez semejante, pero completamente distinto e independiente, del proceso que lo produjo en el gambá acuático. Las convergencias evolutivas no son más que el efecto de superficie de procesos independientes de divergencia a partir de formas ancestrales diferentes; y lo que la Teoría de la Selección Natural realmente explica son esos procesos de divergencia. Aquí, igual que en los casos en donde ninguna convergencia es verificada o conocida, la verdadera pregunta es ipor qué la especie $X$ tiene un rasgo ausente en su forma ancestral Y?; y por eso se puede también decir que el ideal de orden natural que le da sentido a esta pregunta es esa presunción de permanencia de las formas a la que aquí se ha aludido. La pregunta clave siempre es: ipor qué la diferencia y no más bien la semejanza?; ipor qué el cambio y no la permanencia?; o incluso: ¿por qué la apomorfia y no más bien la plesiomorfia?

Claro, una cosa es la mera explicación de las analogías por convergencia adaptativa; y otra cosa totalmente distinta es la explicación de las genuinas homologías. Es decir: la explicación de la verdadera unidad de tipo. En el darwinismo, se dice, éstas se explican por filiación común (Darwin 1859, p. 206); y esto también parece ir en contra de la alegación de que el objetivo explicativo de la teoría darwiniana se encuentra en las diferencias, y nunca en las semejanzas, entre los seres vivos. El uso generalizado de esa regla de inferencia que Sober (1999, p. 253) llamó Modus Darwin ("semejanza, luego ascendencia común”) no parece indicar otra cosa. ¿Por qué los yacarés y los cocodrilos se parecen? ¿Tal vez porqué su modo de vida es semejante y entonces están sometidos a presiones selectivas similares? No, antes que eso, ellos se parecen porque derivan de un ancestral común; y son en todo caso las diferencias en sus modos de vida las que, selección natural mediante, explicarán las diferencias morfológicas que ellos presentan.

El modo de vida, como vimos en el caso de las membranas interdigitales de las nutrias y gambás d'agua, puede eventualmente producir esta semejanza. Pero lo que en principio se da por supuesto, hasta que sea desmentido por evidencia adicional, es que la semejanza obedece a la filiación común; y el hecho de que, a priori, se prefiera la explicación por filiación común, por Modus Darwin, a la 
explicación por convergencia o reversión (ver Tassy 1998, p. 250), es altamente significativo. El Modus Darwin sólo puede funcionar como regla de inferencia, y no como mera generalización empírica, en un mundo donde la permanencia de la forma originaria o inicial es considerada como el grado de cero de cualquier explicación. Todo lo demás, todo lo que ocurra por sobre ese horizonte de permanencia, será explicado por selección natural. Atribuir la semejanza al origen común es siempre la alternativa menos problemática no por ser la hipótesis más segura sino por ser la hipótesis mínima; y esto se torna evidente en la sistemática filogenética. Los seguidores de Hennig (1968) aplican en sus análisis un principio de parsimonia que está estrechamente relacionado con esa parsimonia ontológica que, según dije, funciona como ideal de orden natural de la teoría darwiniana.

En cierto sentido, la parsimonia cladista puede ser vista como una extensión al dominio de los estudios filogenéticos de un principio general de simplicidad o de economía de hipótesis que Newton ([1726] 1962, p. 398) propuso en sus Principia, y como primera regla del razonamiento filosófico: no admitir más causas de las cosas naturales que aquellas que son suficientes para explicar su aparición. En cualquier dominio de experiencia, esta regla nos lleva, en efecto, a preferir las explicaciones o reconstrucciones de procesos que minimicen tanto las causas involucradas como las etapas seguidas (Sober 1994, p. 170); y es a eso que aluden los cladistas cuando nos dicen que, a la hora de elegir entre reconstrucciones filogenéticas alternativas, debemos preferir hipótesis o sistemas de hipótesis más simples por sobre hipótesis o sistemas de hipótesis más complejos (Eldredge \& Cracraft 1980, p. 67, Wiley 1981, p. 111).

Pero lo interesante, lo que aquí no puede dejar de llamarnos la atención y aquello que no podemos evitar relacionar con la tesis aquí sostenida sobre el ideal de orden natural de la Teoría de la Selección Natural, es el hecho de que allí se entienda que mayor simplicidad sea lo mismo que minimización de los cambios evolutivos. El criterio de simplicidad, como observa Pascal Tassy (1991, p. 55), remite en este contexto a la economía de hipótesis evolutivas: "A partir de un número dado de observaciones tanto de fósiles cuanto de formas actuales, procuramos construir para las especies o grupos de especies estudiados un ordenamiento que implique el mínimo de eventos evolutivos". La idea general es que "una solución que implique el mínimo de eventos evolutivos no puede ser menos probable que una solución que implique más” (Tassy 1991, p. 55).

En efecto, según la perspectiva cladista, "la hipótesis más parsimoniosa sobre un árbol evolutivo es aquella que requiere la menor cantidad posible de cambio evolutivo" (Sterelny \& Griffiths 1999, p. 200). Es decir: el árbol filogenético más corto, él más parsimonioso, es el "árbol que contiene el número más pequeño de pasos evolutivos" (Tassy 1998, p. 251); y, sobre todo, "es aquel en donde cada uno de los rasgos aparece sólo una vez; o a falta de él, es aquel donde cada uno de los rasgos aparece el mínimo de veces" (Tassy 1998, p. 258). El árbol filogenético más parsimonioso es, en suma, aquél que supone menos hechos evolutivos a ser explicados; y los hechos evolutivos no son otra cosa que eventos de divergencia a partir de una forma ancestral. Con todo, y más allá del aire de familia que ese principio metodológico de parsimonia guarda con la parsimonia ontológica del ideal darwiniano de orden natural, debemos cuidarnos de no confundir ambas ideas: ni la parsimonia ontológica darwiniana es una simple hipóstasis de la parsimonia metodológica cladista; ni esta última se deriva inmediatamente de aquélla.

Del mismo modo en que el compromiso con el ideal de orden natural de la física clásica no nos lleva a pensar en un mundo donde las aceleraciones y desaceleraciones sean fenómenos ausentes o poco comunes, el compromiso con el ideal de orden natural darwiniano no nos lleva, de por sí solo, a pensar que los eventos evolutivos sean pocos; lo que él hace es decirnos que tales fenómenos precisan explicación. Y es ahí donde reside, en nuestra opinión, la diferencia más clara entre ambos principios. Es que, a diferencia de un ideal de orden natural, el principio de parsimonia de los cladistas no sirve para definir qué es lo que podría considerarse un hecho a ser explicado. En lugar de ello, este principio opera como una regla metodológica que nos orienta respecto de qué tipo de reconstrucciones debemos preferir para cierto tipo de procesos, siendo que la recomendación que el mismo nos da es justamente la de preferir aquellas reconstrucciones que minimizan aquellos fenómenos que el ideal darwiniano de 
orden natural nos propone como dignos de explicación. Pero no nos apuremos ahora a ver una contradicción o una paradoja donde no hay más que una estrecha complementación.

El hecho de que se considere la explicación de la semejanza por filiación común como más económica, o más simple, que una explicación que apele a una multiplicación de las transformaciones, nos está indicando que se considera que la permanencia de las formas ancestrales constituye un fenómeno que requiere una inversión o un esfuerzo explicativo menor que el requerido por las propias transformaciones evolutivas. Sin entrar en la polémica de hasta donde la parsimonia cladista precisa ser justificada por presunciones o hipótesis sobre el fenómeno evolutivo (ver Sober 1988, p. 198, 1993, p. 179, Tassy 1991, p. 56, 1998, p. 267), creo que la propia idea de simplicidad que Hennig y sus seguidores invocan supone la permanencia de la forma primitiva como ideal de orden natural. Si lo que cuenta como hecho a ser explicado y constatado es siempre la divergencia de las formas a partir de un estado inicial cualquiera; entonces, las reconstrucciones filogenéticas que apelan al menor número de tales divergencias, minimizan, al mismo tiempo, la cantidad de hechos presupuestos y posteriormente necesitados de explicación. Menos divergencias implican menos pasos evolutivos a ser introducidos en nuestra reconstrucción; y esto da como resultado hipótesis o reconstrucciones más simples.

La recomendación de preferir una hipótesis simple a una hipótesis compleja, sobre todo si es enunciada con la generalidad que Newton le concede en los Principia, puede ser, tal vez, justificada por consideraciones generales, de carácter metodológico u ontológico, que no apelen ni explicita ni implícitamente a nuestra concepción del fenómeno evolutivo. Pero, al identificarse esa economía con la minimización de las hipótesis que aludan a pasos evolutivos, se está presuponiendo que la ausencia de tales eventos, la permanencia de la estructura que remite al tipo ancestral, es algo que no requiere explicación: eso es lo que cabe esperar que de por sí ocurra. Suponer esa permanencia parece ser algo muy próximo a no suponer nada: parece ser la simple, la inocente e incuestionable aceptación de lo obvio.

El valor epistemológico de la simplicidad puede ser general; y podemos argumentar en su favor en base a consideraciones metodológicas de diversa índole o en base a supuestos metafísicos sobre la simplicidad ultima del mundo. Pero lo que hemos de entender por simplicidad depende de contextos teóricos específicos: no hay una idea general y universal de simplicidad; sino nociones regionales sujetas a los avatares de la historia de la ciencia. De nada nos sirve la exigencia de austeridad si no tenemos criterios o escalas de valor para realizar cálculos de costos; y esos criterios, tanto en la ciencia como en la economía, cambian conforme los momentos y las situaciones. Así, la presunción cladista de que la simplicidad equivale a menos divergencias y transformaciones evolutivas, depende de ese ideal de orden natural específico de la Teoría de la Selección Natural que es la permanencia de la forma primitiva; y hasta me permito ir un poco más lejos: en todo contexto teórico la noción de simplicidad depende del ideal de orden natural que esté en vigencia.

Como sugieren Sterelny y Griffiths, la presunción empíricamente contrastable de que "el cambio es raro en comparación al no cambio" (Sterelny \& Griffiths 1999, p. 200) puede servir de soporte adicional y de motivación para la parsimonia metodológica cladista: la parsimonia de la evolución, el carácter preponderantemente conservador o estabilizador de la selección natural, parece invitarnos a esa economía de hipótesis evolutivas recomendada por Hennig; pero esta última actitud reposa ya antes en esa parsimonia ontológica darwiniana a la cual estamos aludiendo. El criterio cladista de economía puede justificarse independientemente de nuestro conocimiento del curso efectivo de la evolución; pero aun así depende de un ideal de orden natural que es propio de la Teoría de la Selección Natural. La parsimonia cladista no es teóricamente neutral, es una parsimonia darwiniana. $\mathrm{O}$ dicho con mayor precisión: es una parsimonia metodológica sustentada en una presunción de parsimonia ontológica específicamente darwinista; y su adopción pone en evidencia el ideal de orden natural sobre el que se recortan los hechos a ser explicados por la Teoría de la Selección Natural. 


\section{Hay más fuerzas, hay otras cosas a explicar}

Pero si la Sistemática Filogenética parece sujetarse al mismo ideal de orden natural de la Teoría de la Selección Natural, la Biología Evolucionaria Desenvolvimiental parece no hacerlo. En ella, la diversidad de las formas parece ser algo mucho menos interesante, menos urgente de ser explicado, que el hecho de que esa misma diversidad sea siempre limitada; y es ahí en donde puede comenzar a entreverse la entrada en juego de un ideal de orden natural distinto de aquel sobre el que se funda la Teoría de la Selección Natural. Con todo, para entender realmente en qué sentido se pude afirmar que la filogenia despliega un abanico de formas más restricto que lo esperable es necesario analizar más de cerca la acción de esas fuerzas evolutivas que algunos teóricos de la Evo-Devo llaman de constreñimientos desenvolvimientales. Si es cierto que alli hay en juego otro ideal de orden natural, ellos son las fuerzas llamadas a explicar los desvíos que de él se produzcan; y es por eso que para individualizarlo correctamente tenemos que comprender el modo en que dichas fuerzas operan.

Estos constreñimientos desenvolvimientales, concomitantemente con otros factores como la selección natural, pautan el curso de la evolución, permitiendo explicar la dirección y la secuencia de los cambios evolutivos (ver Hall 1992, p. 7, Sterelny 2000, S374, Gould 2002, p. 1028, Wilkins 2002, p. 384, Laubichler 2007, p. 347); y es para rescatar el carácter positivo, y no meramente limitativo, de esos factores ontogenéticos que Wallace Arthur (2004a, p. 16, 2004b, p. 283) propone el término "sesgo desenvolvimiental" [developmental bias]. El mismo englobaría tanto las limitaciones, los genuinos constreñimientos [constraints], como los verdaderos direccionamientos [drives] que los procesos ontogenéticos le imponen a los fenómenos evolutivos. Y como es ese sesgo el que produce el desvío del orden natural que la Biología Evolucionaria Desenvolvimiental quiere explicar, conocer la índole de sus efectos constituye el mejor camino para individualizar y comprender la naturaleza de dicho orden.

Para entender ese sesgo, largamente ignorado por la ortodoxia neodarwiniana, debemos pensar en lo siguiente: toda innovación evolutiva posible, toda alternativa que pueda ofrecerse al escrutinio de la selección natural, tiene que poder corporizarse antes en una alteración ontogenética viable (Amundson 2001, p. 314, Schwenk \& Wagner 2003, p. 59, Laubichler 2007, p. 343). Para que una variación fenotípica surja y pueda entrar en competencia darwiniana con otras, algo en el proceso de la ontogénesis tiene que ser atrofiado o hipertrofiado, agregado o suprimido, transpuesto o deformado, postergado o anticipado; y es ahí en donde encuentra su relevancia aquello que Ron Amundson ha llamado "Principio de Completud Causal": "Para producir una modificación en la forma adulta, la evolución debe modificar el proceso embriológico responsable por esa forma. Por eso, para comprender la evolución es necesario comprender el desarrollo" (Amundson 2005, p. 176).

Sea cual sea la índole de la reprogramación (Arthur 2000) de la ontogenia producida por la variación, ella tiene que cumplir con dos requisitos fundamentales. En primer lugar, tiene que ser accesible para el sistema en desarrollo (Maynard Smith et al. 1985, p. 269, Raff 2000, p. 78), es decir: tiene que tratarse de una alteración pasible de ser producida, con relativa facilidad, en y por ese mismo proceso ontogénetico (Arthur 1997, p. 48, Azkonobieta 2005, p. 118); y, en segundo lugar, tiene que ser tal que, ni aborte ese proceso, ni genere un monstruo totalmente inviable (Amundson, 2001, p. 320). Además de física o fisiológicamente posible, un cambio evolutivo tiene que ser ontogenéticamente posible e, incluso, probable (ver Amundson 2005, p. 231, Azkonobieta 2005, p. 118).

La ontogénesis puede o no recapitular a la filogénesis; pero con seguridad la limita y la orienta (ver Hall 1992, p. 11, Wilkins 2002, p. 384). La limita estableciendo cuáles modificaciones son viables y cuáles no; pero al hacer eso también la orienta: si un rasgo A puede cambiar a la forma $A^{\prime}$ o a la forma A", pero la viabilidad de $A^{\prime}$ depende de que simultáneamente a ella se dé otra serie compleja de cambios en otros rasgos, y la viabilidad de A" no depende de esa coincidencia feliz; entonces éste será un cambio más probable que aquél. Para la evolución, para decirlo de algún modo, el estado $A$ " será más accesible, más fácil (ver Arthur 2004b, p. 283), que el estado A'; y esto puede explicar que A" se dé, y no $A^{\prime}$, aun cuando nosotros pudiésemos imaginar que éste sería darwinianamente más eficaz que 
aquél. Una innovación puede ser muy útil; pero si ésta es muy difícil de ser incorporada al proceso de desarrollo, entonces es muy posible que nunca ocurra y que en su lugar sí ocurra otra innovación, tal vez un poco menos eficaz en términos adaptativos, pero que exige una reformulación menor y resulte menos improbable desde la ontogénesis.

La selección natural, ya lo sabemos, siempre opera sobre una oferta previa de alternativas viables (si no fuesen viables no podrían entrar en competición y no cabría hablar de selección natural); y el estudio de los constreñimientos ontogenéticos puede permitirnos explicar la composición de esa oferta (Amundson 1998, p. 108, 2001, p. 326). Un constreñimiento desenvolvimiental, tal como ya fue definido en el consensus paper organizado por Maynard Smith, Dick Burian y Stuart Kauffman en 1985, sería justamente "un sesgo en la producción de variantes fenotípicas o una limitación de la variabilidad fenotípica, causada por la estructura, carácter, composición, o dinámica del sistema desenvolvimiental" (Maynard Smith et al. 1985, p. 266); y ese sesgo, obviamente, definiría el margen de juego de la selección natural. En cierta forma esto parece lo de siempre: la variación propone y la selección natural dispone; pero ésta sólo dispone dentro de un abanico restricto de alternativas que aquella insiste en proponer (ver West-Eberhard 2003, p. 25, Arthur 2004a, pp. 131, 195).

Decir, entonces, que la selección natural es la única fuerza que, en ese caso, está guiando la evolución sería como creer que cuando optamos entre uno de los dos únicos caminos posibles para llegar a otra ciudad, somos nosotros los que estamos trazando la ruta. O peor: sería como creer que cuando el voto popular consagra uno entre dos candidatos a presidentes, son los ciudadanos los que están pautando el destino de la república. No se trata, claro, de decretar que Brian Goodwin tenga razón y que la selección natural no sea más que una ilusión semejante a la que produce navegar en un barquito de Disneylandia (ver Dennett 2000, p. 338); pero se trata de aceptar la posibilidad de que la evolución, a la manera de los barcos del Mississippi de Mark Twain, deba restringirse a ciertos canales fuera de los cuales la propia navegación se hace imposible.

Pero, además de permitirnos explicar la oferta de variaciones que pueden darse en una determinada población, el estudio de los procesos de desarrollo también puede permitirnos entender la secuencia de las innovaciones evolutivas. La ontogénesis es un proceso necesariamente secuencial: una estructura sólo surge cuando existen otras estructuras previas que le sirven de base; y el estudio de esas etapas puede permitirnos determinar cuáles fueron los pasos que siguió la propia evolución. Si en la ontogénesis, un órgano A surge por la diferenciación de las células que componen el tejido de un órgano $B$ ya parcialmente conformado; entonces, podemos inferir que $A$ es una innovación evolutiva posterior a $B$. La filogénesis puede hacer muchas cosas con $A$ y con $B$; pero si las células de $A$ son una especialización de las células de $B$, ella necesariamente tuvo que producir a $B$ antes que a $A$. La ontogénesis, lo vemos otra vez, pauta y ordena los pasos de la filogénesis (Hall 1992, p. 11, Amundson 2005, p. 90); y este aspecto secuencial es clave para entender la senda efectivamente seguida por la evolución. La paleontología, es cierto, puede mostrarnos cuál fue de hecho esa secuencia; pero su razón última quizá sólo se explique por esa pauta que la ontogenia le impone a la filogenia.

No todo es posible, parece constatar el teórico de la Evo-Devo: los requerimientos de la ontogenia limitan las posibilidades de la evolución. Hay formas que ellas nunca podrá producir porque hacerlo supondría desatender esas exigencias; y, en este sentido preciso y limitado, puede decirse que el programa y los objetivos de la Biología Evolucionaria Desenvolvimiental guardan cierta analogía con el programa y los objetivos de la anatómica comparada cuvieriana. Es que Cuvier, como observó Jordi Agustí (2003, p. 54), tenía "una visión arquitectural del ser vivo"; y su primera constatación fue "la existencia de arquitecturas prohibidas": combinaciones concebibles de órganos que, sin embargo, estaban excluidas de la naturaleza. Nuestra imaginación puede, en efecto, figurarse un monstruoso carnívoro que, a sus colmillos, agregue cuernos y pesuñas. La naturaleza, sin embargo, no puede generarlo: un ser semejante no es posible, no tiene condición de existencia; y no la tiene porque su constitución repugna a ciertos principios fundamentales que establecen el dominio de los seres posibles. Es objetivo de la anatomía comparada el poder conocer esas leyes de la correspondencia entre 
órganos que, al mismo tiempo, definirán el universo de los seres posibles y nos explicarán por qué es que ciertas combinaciones de órganos están ausentes de la naturaleza.

Pero, si a Cuvier le importaba entender por qué no era posible un carnívoro con cuernos, a los teóricos de la Evo-Devo les interesa saber por qué no hay vertebrados hexápodos (Raff 1996, p. 295, Sterelny \& Griffiths 1999, p. 232, Arthur 2004a, p. 9); y mientras Cuvier (1805 p. 58) quería explicar esas limitaciones en base a leyes de la correlación de los órganos que definirían el universo de las organizaciones fisiológicas posibles, los teóricos de la Evo-Devo las quieren explicar, sea en base a constreñimientos ontogenéticos que limitarían el universo de las innovaciones morfológicas que pueden ocurrir en un momento dado de la historia evolutiva de un taxón (Amundson 2005, p. 230), sea en base a los direccionamientos desenvolvimientales que empujan a la evolución por ciertas sendas privilegiadas (Arthur 2004b, p. 283). Por otro lado, y del mismo modo en que Cuvier estaba interesado en mostrar que esas limitaciones también permitían entender el tipo de organización de las formas efectivamente presentes en la naturaleza, a los teóricos de la Evo-Devo les interesa mostrar cómo el árbol de la vida puede generarse dentro de ese conjunto de restricciones que los constreñimientos ontogenéticos le imponen a la filogenia.

De ahí deriva el interés que para ellos revisten las grandes invenciones o novedades evolutivas: el surgimiento de nuevos y diferentes planes corporales, como el de los artrópodos o vertebrados, el surgimiento de seres modulares o segmentados, o la aparición de vertebrados dotados de miembros, son cosas que sorprenden y piden por explicación, porque, inmediatamente, ellas son proyectadas sobre un conjunto de restricciones que, se supone, deben ser sorteadas o dribladas, pero nunca anuladas (ver Laubichler 2007, pp. 354-355). En cierto sentido, y como ya lo dijo Kim Sterelny (2000, S375), "La novedad es simplemente lo inverso del constreñimiento". Pero, vistas desde un ángulo ligeramente diferente, esas invenciones, esos quiebres de las homologías (Laubichler 2007, p. 355), también son importantes porque, si se asume que existen direccionamientos desenvolvimientales que llevan a la evolución por ciertos caminos antes que por otros, también será necesario saber cuáles fueron los direccionamientos que empujaron en la dirección de esa innovación. De todos modos, en uno y otro caso, la pregunta ya no es exactamente ¿por qué no todo es posible? o ipor qué algunas cambios son más probables que otros?; sino más bien ¿cómo fue esto posible?

Pero esta segunda pregunta se plantea porque previamente se ha asumido que esas invenciones, no sólo surgen sobre un horizonte de constreñimientos y limitaciones que deben superarse, sino que además, para ser alcanzadas, las mismas deben estar en la trayectoria de ese direccionamiento que la ontogenia le impone a la oferta de variaciones. Si algo ocurre, en síntesis, es porque es posible; y para comprender esa posibilidad hay que mostrar cómo es que ella se encaja en ese universo de restricciones y de direccionamientos que, se supone, limitan y pautan la senda de la evolución. Para que la posibilidad de algo se presente como un hecho problemático que requiere explicación, esa posibilidad tiene que ser considerada bajo la presunción de que no todo es posible. Si el universo de las formas posibles y accesibles al cambio evolutivo no fuese percibido como restringido, la pregunta por la posibilidad de las invenciones evolutivas no tendría mayor importancia. Ellas serían percibidas y explicadas sólo a la manera de la Teoría de la Selección Natural; es decir: como meros alejamientos del tipo ancestral exigidos por los avatares de la lucha por la existencia.

La pregunta no sería entonces ¿cómo fue ese cambio posible?; sino que sería ¿por qué es que ese cambio, ese alejamiento de la forma ancestral, fue conveniente? Pero, por su parte, la idea de que no todo es posible sólo tiene sentido si el universo de lo actual, el repertorio de las formas realmente existentes, es pensado como un recorte de un universo más amplio de alternativas concebibles, algunas de las cuales son realizables y otras no. Es decir: esas mismas restricciones que hacen tan interesantes a las grandes innovaciones evolutivas sólo pueden entrar en consideración, sólo pueden ser constatadas, en la medida en que ellas contradicen la presunción de un mundo más rico que el efectivamente producido por la evolución. Sin esta presunción las limitaciones de lo posible evolutivo no serían percibidas como tales; y si ellas no fuesen percibidas, las grandes invenciones evolutivas no revestirían mayor interés. 
Éstas sólo serían consideradas como respuestas a presiones selectivas; y nunca seríamos llevados a preguntarnos por cómo pudieron ser ontogenéticamente posibles y accesibles.

Es claro, además, que esas innovaciones también resultan interesantes porque nos enseñan algo sobre esos mismos constreñimientos y direccionamientos a las que ellas deben ajustarse. Del mismo modo en que para Cuvier cada especie biológica particular constituye un teorema que confirma y permite entender las leyes de la anatomía comparada, para los teóricos de la Evo-Devo, cada innovación evolutiva supone, y pone en evidencia, ese sesgo desenvolvimiental que pauta el curso de la evolución y define el margen de maniobra de la selección natural; y por eso también puede decirse que las innovaciones morfológicas son como experimentos naturales que nos permiten entender por qué la oferta de variaciones y de alternativas sobre la cual habrá de trabajar la selección natural es menos amplia de lo que hubiésemos pensado. Esas innovaciones, en suma, pueden ser la mejor clave para responder el enigma fundamental de la Evo-Devo: ipor qué no todo es posible? Un enigma que sólo parece tener sentido en el marco de un contraste entre algo así como una plenitud presumida y esa relativa pobreza de formas que exhibe lo real.

\section{4. ¿De qué morfoespacio hablamos?}

Cuidémonos, sin embargo, de incurrir en el error de pensar que ese descubrimiento de las limitaciones de lo posible tenga como telón de fondo una supuesta presunción darwiniana de plenitud. Además del hecho de estar basada en un ideal de orden natural que niega esa plenitud, la Teoría de la Selección Natural implica, por sí misma, predicciones restrictivas sobre el universo de formas que la evolución puede producir. La más obvia y célebre es aquella según la cual la selección natural no puede "modificar la estructura de una especie [...] para el bien de otra especie” (Darwin 1859, p. 87). Pero existe otra todavía más fundamental: la selección natural sólo puede producir modificaciones que incrementen, de un modo directo o indirecto, como en el caso de la selección parental, el éxito reproductivo diferencial de sus portadores; y esto limita mucho la capacidad de la selección natural para descubrir soluciones a los diferentes problemas adaptativos que una población debe resolver. Una serie de cambios morfológicos, cuyos diferentes eslabones no implique alguna ventaja para sus portadores, no puede ser producida por la selección natural; aun cuando el resultado final pudiese parecer altamente ventajoso y adaptativo.

Por otro lado, aunque el universo de las presiones selectivas a las que están sometidas las diversas formas de vida sea terriblemente variado y heterogéneo, jamás podríamos decir que la selección natural se aproxime a una saturación del universo de formas que ella sería capaz de producir. Hay muchas herejías morfológicas que aún no fueron ensayadas, y tal vez nunca sean ensayadas, porque simplemente nunca se presentó, y tal vez nunca se presente, una oportunidad en donde sea ventajoso producirlas. Lejos de tender a generar todas las formas posibles, la selección natural se limita a producir las que, en algún momento, presenten alguna conveniencia detectable, como ya dije, en términos de éxito reproductivo individual; y por eso sería ingenuo considerar sus realizaciones como si fuesen un catálogo completo de las morfologías posibles. Por fin, e independientemente del hecho de que esto no siempre sea recordado en las exposiciones y, quizá, en algunas aplicaciones de la Teoría de la Selección Natural, es necesario también apuntar que ésta no presupone, en sí misma, ninguna actitud ingenua al respecto de los constreñimientos físicos, fisiológicos y, eventualmente, desenvolvimientales que limitan lo biológicamente posible.

Partiendo de la base de que la selección natural es siempre una opción entre alternativas efectivamente existentes, y no entre alternativas potenciales, los darwinistas tendieron a desentenderse, tal vez por el error de considerar que se trataba de algo obvio, de los factores que limitaban la oferta de tales alternativas. Pero eso no significa que la Teoría de la Selección Natural suponga, o precise suponer, la inexistencia de dichos factores. Decir que el darwinismo se basa en el supuesto de que "los organismos pueden tomar cualquier forma" (Goodwin 1998, p. 112), o que, según él, "un número indefinido de formas es posible" (Webster 1996, p. 103) es confundir la estructura conceptual intrínseca de la Teoría de la Selección Natural con el desconocimiento, o el descuido, que algunos 
darwinistas tuvieron con relación a ciertas temáticas que tal vez estaban demasiado lejos del foco de sus investigaciones y debates; y esta confusión en nada contribuye, claro, para una correcta comprensión de la relación existente entre dicha teoría y la Biología Evolucionaria Desenvolvimiental.

Acusar al darwinismo de no percibir que existen límites físicos para lo biológicamente posible sería, como el propio Goodwin (1998, p. 112) lo reconoce, algo así como vapulear un espantapájaros que nadie se tomó el trabajo de armar. Pero eso también vale para la relación entre lo evolutivamente posible y fisiológicamente posible: por definición, la selección natural sólo puede trabajar a partir de aquello que, fisiológicamente hablando, se sostiene. No es que los organismos incapaces de cumplir con sus funciones fisiológicas más básicas se salgan mal en la lucha por la existencia: ellos están a priori excluidos de dicha lucha. Y esto último se extiende, casi de inmediato, a lo desenvolvimientalmente posible: la selección natural sólo trabaja sobre aquello que le ofrecen los procesos ontogenéticos. Sobre esta oferta ella hace sus opciones; y por eso, querer definir lo desenvolvimientalmente posible a partir de lo darwinianamente posible es invertir el orden del razonamiento y el orden de las cosas. Es como querer definir lo físicamente posible a partir de lo biológicamente posible.

En realidad, lo darwinianamente accesible sólo puede ser un recorte de lo desenvolvimientalmente posible. Por eso, si se quiere dar una definición general de ese morfoespacio más denso, o más frondoso, que, según dije en la presentación, sirve como ideal de orden natural de la Biología Evolucionaria Desenvolvimiental, si se quiere caracterizar y acotar significativamente ese universo de formas biológicamente concebibles, esa Biblioteca de Mendel (Dennett 1995, p. 107) o ese "gigantesco museo de la imaginación zoológica" (Dawkins 1996, p. 182), del cual lo desenvolvimientalmente posible sería un subconjunto particular, será necesario mirar en otra dirección; y creo que la clave está en el enigma del vertebrado hexápodo al cual apeló Rudolf Raff (1996, p. 295) para explicar el concepto de constraint. Pero, para que él nos sirva, será necesario hacer un uso de ese ejemplo ligeramente distinto de aquel propuesto por Raff.

Es que, al igual que Wallace Arthur (2004a, p. 10) en Biased Embryos and Evolution, Raff lo usa en el contexto de un contraste entre aquello que podría llegar a ser darwinianamente ventajoso y aquello que resulta desenvolvimientalmente posible. Raff y Arthur sugieren, en efecto, que un vertebrado con seis patas podría tener muchas ventajas, pero apuntan que, no obstante eso, la evolución nunca lo produjo; y la explicación de esa imposibilidad estaría en que "ciertos constreñimientos desenvolvimientales podrían tornar imposible que un embrión de vertebrado genere más de dos pares de miembros" (Raff 1996, p. 295). Es decir: ambos autores parecen incurrir en el equívoco de pensar lo ontogenéticamante posible como si fuese una subclase del espacio, en realidad indefinible, de lo darwinianamente esperable. Pero, aun así, la imagen del vertebrado hexápodo puede ser provechosa. Ella resulta útil por una razón muy simple: independientemente de considerarla o no como darwinianamente ventajosa, esa morfología parece ser funcional o fisiológicamente viable; y esto nos pone ante un contraste, a mi ver, más esclarecedor: aquel que puede establecerse entre lo funcionalmente posible y lo desenvolvimientalmente plausible.

Un vertebrado de seis patas sería ontogeneticamente inviable: hasta donde se sabe, un embrión de tetrápodo no podría generar, o tolerar, las modificaciones que llevarían al desarrollo de un tercer par de miembros realmente funcional para la locomoción. Sin embargo, y por lo menos en la medida en que podemos establecer una distinción de razón entre la ontogenia y el funcionamiento del organismo constituido, todo parece indicar que un ser con esas características sería fisiológicamente posible. Un ser semejante podría cumplir sus funciones básicas y gozar, por lo menos, de una mínima viabilidad ecológica aunque sea en medios muy restrictos. Pero, si este ejemplo nos parece demasiado dudoso y conjetural, podemos ilustrar la distinción entre lo funcional y lo desenvolvimientalmente posible en base a fenómenos más conocidos y habituales.

Los transplantes heterotópicos y los alotransplantes permiten producir morfologías funcionales, tales como un hombre con dos corazones, o con un páncreas en la pelvis, o con un hígado de cerdo. Formas, todas estas, que difícilmente serían generadas por una reprogramación desenvolvimiental; y algo semejante podría tal vez decirse de muchas mutilaciones y amputaciones que los heridos de guerra deben 
soportar: ellas generan morfologías que, en general, rompen con las simetrías y las regularidades a las que se ajustan, incluso, los procesos teratológicos; pero, aun así, los individuos que las padecen consiguen cumplir con sus funciones fisiológicas fundamentales. Los organismos adultos parecen gozar de una modularidad que permite que en ellos sean producidas modificaciones puntuales -agregados, substracciones o transposiciones- que un proceso de desarrollo normal nunca produciría; o, por lo menos, nunca produciría sin también alterar otras estructuras que, en el caso de los transplantados y mutilados, permanecen iguales. Y a estos ejemplos se podrían agregar los de las quimeras producidas por el injerto de dos embriones de especies distintas como cabra y oveja.

En estos últimos casos, es cierto, hay un proceso de desarrollo que continúa a partir de ese injerto: éste, podríamos decir, produce una reprogramación viable de la ontogenia; pero lo hace a partir de un punto, el propio injerto, que ninguna reprogramación ontogenética podría producir. Ese injerto es, por eso, un estado funcionalmente viable pero ontogenéticamente inaccesible; y esto no constituye ninguna rareza. Es sólo un ejemplo más extremo de lo que ocurriría con un recién nacido al cual se le transplantase un segundo corazón: su desarrollo continuaría, pero lo haría a partir de un estado inicial que resulta, él mismo, ontogenéticamente inaccesible.

El espacio de lo funcionalmente posible, que Cuvier pretendía determinar a partir de las leyes de la correlación de los órganos, es, en efecto, más restringido que el espacio de lo físicamente posible; pero, resulta más amplio que el de lo desenvolvimientalmente accesible. En el espacio de lo funcionalmente viable hay algunas formas y algunas combinaciones de órganos que ningún proceso o reprogramación desenvolvimiental podría generar; y por eso, aun cuando algunas de esas formas y combinaciones puedan parecernos adaptativamente ventajosas en ciertos contextos, la selección natural jamás nos sacará de la duda sobre si realmente lo son, porque ellas nunca se ofrecerán a su escrutinio.

Puede decirse, en síntesis, que el espacio de las morfologías funcionalmente posibles es más denso que el espacio de las morfologías desenvolvimientalmente generables o desenvolvimientalmente aceptables. Dado cualquier tipo de organismo, el conjunto de modificaciones que los mismos podrían tolerar en cualquier etapa de su desarrollo es mayor que el conjunto de modificaciones que son ontogenéticamente accesibles y mayor que el conjunto de modificaciones que, en caso de ser alcanzadas, permitirían que esa ontogénesis continúe y llegue a buen término. Es decir: dado el conjunto de las reprogramaciones viables de la secuencia de etapas ontogenéticas $A \rightarrow B \rightarrow C$ de un organismo $X$, habrá siempre dos conjuntos de estados modificados de $B$ y $C$ que serían funcionalmente posibles para $X$ pero inaccesibles desde $A$ o desde $B$; y habrá también un conjunto de estados modificados de $B$ que, aun siendo funcionalmente viables y accesibles desde $A$, no darían lugar a ninguna etapa subsiguiente de la ontogénesis.

El espacio de lo funcionalmente posible puede pensarse como un conjunto de posiciones que representan todas las conformaciones orgánicas fisiológicamente viables. Algunas de esas posiciones, sin embargo, resultan absolutamente inaccesibles para cualquier proceso natural de desarrollo. Las otras, mientras tanto, sólo son accesibles, con mayor o menor facilidad, desde algunas posiciones particulares de ese mismo espacio; y, dentro de este segundo conjunto puede distinguirse otro que estaría compuesto por aquellas posiciones que, en caso de ser alcanzadas, impedirían el pasaje a cualquier otra posición. Se trataría de estados letales o finales para el sistema en desenvolvimiento. Se puede imaginar así una suerte de mapa que marcaría las posibles trayectorias desenvolvimientales que se pueden recorrer a partir de una posición determinada dentro de ese espacio. Algunas de esas trayectorias, claro, serían más fáciles o probables que otras; y algunas resultarían más difíciles.

El estudio de lo que permite, obstruye y empuja cualquier trayectoria entre una serie de posiciones particulares contiguas dentro de ese espacio, será objeto y asunto de la Biología del Desarrollo (ver Arthur 1997, p. 247); pero la reconstrucción del mapa general será el tema de la Biología Evolucionaria Desenvolvimiental. Es decir: la primera nos explicará cómo la ontogenia recorre el trayecto entre dos o más fases diferentes, y funcionalmente viables, del desarrollo tales como lo son dos momentos consecutivos en la metamorfosis de un insecto. Pero la Biología Evolucionaria Desenvolvimiental no sólo será la encargada de reconstruir la serie de reprogramaciones de la ontogenia que, partiendo de la morfología de un reptil, permitieron llegar hasta la morfología de un mamífero; sino que además podrá 
asumir el desafío de mostrar por qué, dada una cierta posición límite dentro de la morfología reptilínea, era más fácil derivar hacia la morfología del proto-mamífero que permanecer en ese lugar de frontera.

Análogamente a una ontogenia particular que recorre un determinado paisaje epigenético, la evolución parece estar condenada y compelida a marchar por ciertos senderos. Pero estos son senderos que pueden bifurcarse y llevarnos hacia atractores muy diferentes de aquellos que guiaban la trayectoria inicial; y el estudio de esas bifurcaciones es justamente el asunto de la Biología Evolucionaria Desenvolvimiental. Dada una forma orgánica cualquiera, el mapa construido por la Biología Evolucionaria Desenvolvimiental, no sólo nos pretende brindar un conocimiento de cuáles serían las sendas de su posible evolución; sino que además quiere permitirnos saber cuáles de ellas son las más probables. Lo primero nos llevará a la temática de los constraints y lo segundo a la del drive. Pero será el conocimiento de ambos aspectos del sesgo desenvolvimiental (ver Arthur 2001, p. 72) lo que permitirá explicar la ocupación sesgada e irregular, tendenciosa podríamos decir, del espacio de lo funcionalmente viable que se producirá a partir de la evolución de esa forma inicial. Y aquí llegamos a un punto fundamental.

La ocupación del espacio de lo funcionalmente posible será siempre parcial aun con independencia de cualquier sesgo o restricción desenvolvimiental. Como ya lo vimos, hay, en primer lugar, morfologías que nunca podrían ser producidas por la selección natural; y hay otras que sólo podrían producirse en circunstancias que, por razones históricas, no se dieron y que tal vez nunca se den. Circunstancias que podrían, o no, ocurrir en el futuro; o que podrían haber ocurrido en el pasado, si las contingencias de la historia de la vida hubiesen sido otras. Hubo un tiempo en que esa provincia del morfoespacio que hoy ocupan los cetáceos estaba vacía; pero hubiese sido un error suponer que la misma era una región prohibida o inaccesible debido a restricciones desenvolvimientales. Y por eso sería también un error suponer que cualquier laguna del morfoespacio constituye un caso, o un asunto, para la Biología Evolucionaria Desenvolvimiental. Lejos de eso, lo que llama a movilizar al sesgo desenvolvimiental como recurso explicativo, es la ocupación irregular de dicho morfoespacio; y esto es lo mismo que decir que la ocupación regular y homogéneamente distribuida del mismo constituye el ideal de orden natural que aquí queremos identificar.

Lo que en la Biología Evolucionaria Desenvolvimiental se busca explicar no es el hecho de que algunas morfologías funcionalmente posibles nunca hayan sido producidas por la evolución: lo que requiere explicación es el hecho de que las que se producen tiendan a concentrarse en ciertas regiones particulares del espacio de lo funcionalmente posible dejando otras contiguas siempre inexploradas; y es eso a lo que se refería Stephen Jay Gould cuando escribía:

La no-homogeneidad del morfoespacio parece tan obviamente intrínseca a la naturaleza (los leones cerca de los tigres, con un gran salto que separa a todos los gatos de los perros y los lobos), que raramente consideramos los problemas que eso plantea. Una vez que la evolución deviene paradigmática, la herencia y la filiación se erigen en la razón más obvia para ordenar las semejanzas reflejadas en nuestras jerarquías taxonómicas. Pero la simple filiación no resuelve todos los problemas suscitados por esos agrupamientos discretos que se dan en el espacio fenotípico; todavía nos queda preguntarnos por qué algunas formas atraen esas concentraciones de diversidad, y por qué existen esos grandes espacios vacíos en algunas regiones, concebibles y no obviamente disfuncionales, del morfoespacio potencial (Gould 2002, p. 347).

Si ese morfoespacio estuviese regular u homogéneamente ocupado, tanto por los seres actuales como por los extintos, no habría nada de particular a ser explicado: allí estaríamos, definitivamente, ante el estado de fuerza cero de esa teoría que los cultores de la Evo-Devo están articulando. Cualquier región de lo funcionalmente posible parecería tan habitable como cualquier otra; y cabría a la selección natural la tarea de explicar por qué dentro de esas regiones se dieron las formas que efectivamente se dieron. Pero, en la medida en que ese morfoespacio se encuentre irregularmente ocupado, con zonas densamente pobladas separadas por grandes intervalos vacíos y siempre inexplorados; entonces habrá que invocar a los contraints y a los drives desenvolvimientales como las causas o fuerzas de ese desvio del orden natural. El problema central de la Evo-Devo no es, por eso, que el mundo de las formas biológicas sea menos rico que lo que hubiésemos podido esperar; su problema es que esa riqueza no sea homogénea, que no sea 
regular; y el sesgo desenvolvimiental es el nombre que cabe darle al conjunto de fuerzas que explicaría esa irregularidad.

Se trata, claro, de un conjunto sumamente heterogéneo. Los constreñimientos y direccionamientos desenvolvimientales que rigen la evolución de los vertebrados deben ser muy distintos a los que rigen la evolución de los insectos; y a ese respecto también deben existir diferencias muy significativas entre mamíferos y batracios. En cada grupo, en cada orden taxonómico, las exigencias de la ontogenia son diferentes. Pero esto no es muy distinto de lo que ocurre con la selección natural: éste no es más que el rótulo de conjunto indefinido y heteróclito de presiones selectivas que actúan de diferente modo sobre diferentes poblaciones; y, por eso, tanto en el dominio de la Teoría de la Selección Natural como en el de la Biología Evolucionaria Desenvolvimiental las fuerzas actuantes en cada situación particular deben ser identificadas inductivamente, caso a caso.

\section{Consideración final}

Lejos del económico y elegante conjunto de leyes fuente que caracteriza a las teorías físicas (ver Sober 1984, p. 51), las teorías de la Biología Evolucionaria funcionan, es decir: explican los hechos que se proponen explicar, en base a un heteróclito y siempre creciente conjunto de mecanismos causales que parece refractario a una presentación unitaria y sistemática; y esto puede desdibujar los perfiles de dichas teorías. Prestar atención a los ideales de orden natural en los que ellas se apoyan, sin embargo, permite que esos contornos se hagan un poco más nítidos. Aunque heterogéneos y difusos, la especificidad de los conjuntos de recursos explicativos que esas teorías movilizan puede entreverse considerando los ideales de orden natural que les dan sentido. Y es esa especificidad la que aquí nos permitió hablar de dos teorías diferentes y relativamente autónomas, pero complementarias, sobre los fenómenos evolutivos: una ya reconocida que es la Teoría de la Selección Natural; y otra ya activa, pero aun no claramente percibida, que es esa Teoría del Sesgo Desenvolvimiental insinuada en las investigaciones y en los resultados de la Evo-Devo.

Bibliografía

Agustí J. (2003), Fósiles, genes y teorías, Barcelona: Tusquets.

Alberch, P. (1989), “The Logic of Monsters: Evidence for Internal Constraint in Development and Evolution”, Geobios (mémoire spécial) 12: 21-57.

Alberch, P. (1980), “Ontogenesis and Morphological Diversification”, American Zoologist 20: 653-667.

Amundson, R. (1998), "Two Concepts of Constraint: Adaptationism and the Challenge from Developmental Biology”, en Hull, D. y M. Ruse (eds.), The Philosophy of Biology, Oxford: Oxford University Press, pp. 93-116.

Amundson, R. (2001), “Adaptation and Development: On the Lack of a Common Ground”, en Orzack, S. y E. Sober (eds.), Adaptationism and Optimality, Cambridge: Cambridge University Press, pp. 303-334.

Amundson, R. (2005), The Changing Role of the Embryo in Evolutionary Thought, Cambridge: Cambridge University Press.

Arthur, W. (1997), The Origin of Animal Body Plans, Cambridge: Cambridge University Press.

Arthur, W. (2000), “The Concept of Developmental Reprogramming and the Quest for an Inclusive Theory of Evolutionary Mechanisms", Evolution $\mathcal{E}$ Development 2: 49-57.

Arthur, W. (2001), “Developmental Drive: An Important Determinant of the Direction of Phenotypic Evolution”, Evolution E Development 3: 271-278.

Arthur, W. (2004a), Biased Embryos and Evolution, Cambridge: Cambridge University Press.

Arthur, W. (2004b), "The Effect of Development on the Direction of Evolution: Toward a Twenty-Century Consensus", Evolution Ë Development 6: 282-288. 
Azkonobieta, T. (2005), Evolución, desarrollo y (auto)organización. Un estudio sobre los principios filosóficos de la Evo-Devo, Tesis doctoral, San Sebastián: Universidad del País Vasco.

Caponi, G. (2004), “La navaja de Darwin”, Ludus Vitalis 12: 9-38.

Cuvier, G. (1805), Leçons d'anatomie comparée, Paris: Badouin.

Darwin, C. (1859), On the Origin of Species, London: Murray.

Dawkins, R. (1996), Climbing Mount Improbable, London: Penguin.

Dennett, D. (1995), Darwin's Dangerous Idea, London: Penguin.

Dennett, D. (2000), "With a Little Help from my Friends”, en Ross, D., Brook, A. y D. Thompson (eds.), Dennett's Philosophy, Cambridge: MIT Press, pp. 327-388.

Eldredge, N. y J. Cracraft (1980), Phylogenetic Patterns and the Evolutionary Process, New York: Columbia University Press.

Fernandez, F., Galliez, M. y M. Leite (2007), “O gambá que inventou o submarino”, Ciência Hoje 39: 70-72.

Goodwin, B. (1998), Las manchas del leopardo, Tusquets: Barcelona.

Gould, S. (1991), "The Disparity of the Burguess Shale Arthropod Fauna and the Limits to Cladistic Analysis: Why we Must strive to Quantify Morphospace”, Paleobiology 17: 411-423.

Gould, S. (2002), The Structure of Evolutionary Theory, Cambridge: Harvard University Press.

Hall, B. (1992), Evolutionary Developmental Biology, London: Chapman \& Hall.

Hennig, W. (1968), Elementos de una sistemática filogenética, Buenos Aires: EUDEBA.

Laubichler, M. (2007), "Evolutionary Developmental Biology”, en Hull, D. y M. Ruse (eds.), The Cambridge Companion to Philosophy of Biology, Cambridge: Cambridge University Press, pp. 342-360.

Maynard Smith, J., Burian, R., Kauffman, S., Alberch, P., Campbell, B., Goodwin, B., Lande, R., Raup, D. y L. Wolpert (1985), "Developmental Constraints and Evolution”, The Quarterly Review of Biology 60: 265-287.

Newton, I. ([1726] 1962), Mathematical Principles of Natural Philosophy, Berkeley: University of California Press.

Raff, R. (2000), “Evo-Devo: The Evolution of a New Discipline”, Nature Reviews Genetics 1: 74-79.

Raff, R. (1996), The Shape of Life: Genes, Development and the Evolution of Animal Form, Chicago: The University of Chicago Press.

Schwnenk, K. y G. Wagner (2003), “Constraint”, en Hall, B. y W. Olson (eds.), Keywords and Concepts in Evolutionary Developmental Biology, Cambridge: Harvard University Press, pp. 52-60.

Schmitt, S. (2006), Aux origines de la biologie moderne, Paris: Belin.

Sober, E. (1984), The Nature of Selection, Chicago: The Chicago University Press.

Sober, E. (1988), Reconstructing the Past: Parsimony, Evolution, and Inference, Cambridge: MIT Press.

Sober, E. (1993), Philosophy of Biology, Oxford: Oxford University Press.

Sober, E. (1994), From a Biological Point of View, Cambridge: Cambridge University Press.

Sober, E. (1999), “Modus Darwin”, Biology EO Philosophy 14: 253-278.

Sterelny, K. (2000), “Development, Evolution, and Adaptation”, Philosophy of Science 67 (Proceedings, Part II): S369. S387.

Sterelny, K. y P. Griffiths (1999), Sex and Death, Chicago: The Chicago University Press.

Tassy, P. (1991), Le message des fossiles, Paris: Hachette.

Tassy, P. (1998), L'arbre a remonter le temps, Paris: Diderot.

Toulmin, S. (1961), Foresight and Understanding, Indianapolis: Indiana University Press.

Webster, G. (1996), “The Problem of Form”, en Webster, G. y B. Goodwin, Form and Transformation, Cambridge: Cambridge University Press, pp. 3-125. 
80 | Gustavo Caponi

West-Eberhard, M. (2003), Developmental Plasticity and Evolution, Oxford: Oxford University Press.

Wiley, E. (1981), Phylogenetics, New York: J. Wiley \& Sons.

Wilkins, A. (2002), The Evolution of Developmental Pathway, Sunderland: Sinauer. 